\title{
FERMI-EDGE SINGULARITY IN LUMINESCENCE SPECTRA OF $p$-TYPE MODULATION DOPED AlGaAs/GaAs QUANTUM WELLS
}

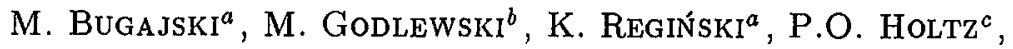 \\ J.P. BERGMAN ${ }^{c}$ AND B. MONEMAR ${ }^{c}$ \\ ${ }^{a}$ Institute of Electron Technology, Al. Lotników 32/46, 02-668 Warsaw, Poland \\ ${ }^{b}$ Institute of Physics, Polish Academy of Sciences \\ Al. Lotników 32/46, 02-668 Warsaw, Poland \\ ${ }^{c}$ Department of Physics and Measurement Technology, Linköping University \\ 58183 Linköping, Sweden
}

We have studied an enhancement of the oscillator strength for optical transitions near the Fermi energy in $p$-type modulation-doped quantum wells, which, so far, deserved much less attention than analogous $n$-type systems, because of the complicated valence band structure involved. The relatively wide ( $L=150 \AA$ ) quantum wells and high doping levels were used, containing more than one occupied subband. The enhancement in the photoluminescence intensity at the Fermi energy resulted from the strong correlation and multiple scattering of holes near the Fermi edge by the localized electrons.

PACS numbers: 42.55.Px, 73.20.Dx

\section{Introduction}

The dynamic response of a hole Fermi sea to the presence of optically generated electrons gives rise to an enhanced interaction of correlated electron-hole pairs near the Fermi level, resulting in an enhanced oscillator strength for optical transitions, referred to as the Fermi-edge singularity (FES). This effect has been studied in detail in $n$-type modulation-doped quantum wells [1-3]. The Fermi-edge singularity or Mahan exciton [4] arises in $n$-type modulation-doped quantum wells from the correlation between a photo-excited hole and the sea of electrons in the quantum well. The only electrons that can contribute to the screening of the positive hole charge are those close to the Fermi surface. So far, $p$-type modulation-doped quantum wells, deserved much less attention than analogous $n$-type systems, because of the complicated valence band structure involved. 


\section{Experimental results}

The investigated samples were high quality $p$-type modulation doped $\mathrm{Al}_{0.3} \mathrm{Ga}_{0.7} \mathrm{As} / \mathrm{GaAs} / \mathrm{Al}_{0.3} \mathrm{Ga}_{0.7} \mathrm{As}$ quantum wells grown by molecular beam epitaxy (MBE) on GaAs substrates. The samples consisted of the $150 \AA$ wells sandwiched between $150 \AA$ thick, doped barriers. The sections of the length of $50 \AA$ separated by $50 \AA$ from the edge of the well were doped with Be. The nominal doping level was: $1 \times 10^{18} \mathrm{~cm}^{-3}$ (\#193.96), $2 \times 10^{18} \mathrm{~cm}^{-3}$ (\#194.96), $4 \times 10^{18} \mathrm{~cm}^{-3}$ (\#195.96) to $8 \times 10^{18} \mathrm{~cm}^{-3}$ (\#196.96).

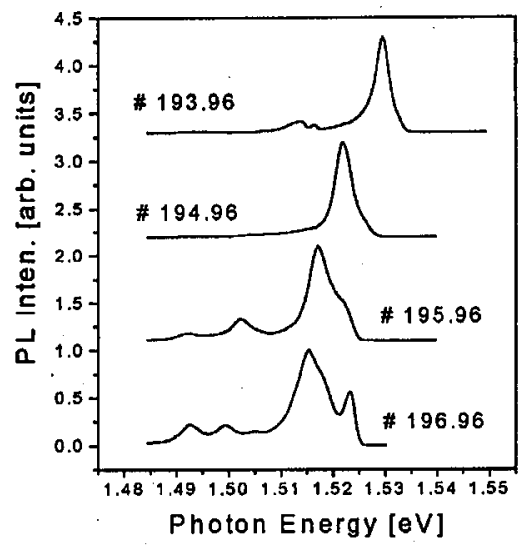

Fig. 1. Photoluminescence spectra of QWs studied, measured at the temperature of $2 \mathrm{~K}$.

In Fig. 1 we show photoluminescence (PL) spectra of the structures studied, recorded at $2 \mathrm{~K}$ and for the excitation with the energy below the AlGaAs barrier band gap energy. For all quantum well (QW) structures the PL spectrum is dominated by the PL peak of the same origin, which shifts down in the energy with an increasing doping level. This dominant PL emission we relate to the recombination transition of photogenerated electrons with 2D free holes accumulated in the GaAs QW regions at the notch potentials at GaAs/AlGaAs interfaces. The red-shift of the $2 \mathrm{D}$ hole $\mathrm{PL}$ is at least partly related to the deepening of the notch potentials confining holes in the GaAs QW regions, which follows an increasing doping level of the structures studied.

The 2D hole PL for the \#196.96 sample, see Fig. 2, shows a weakly resolved structure. A multi-Gaussian fit indicates that this emission consists of two overlapping PL peaks (dashed lines in Fig. 2) separated by about $4.4 \mathrm{meV}$, i.e., by the energy of shallow donors ionization in GaAs. Such value of the PL line-splitting suggests, thus, that 2D free holes recombine either with free or with shallow donor-bound electrons. Donor-related recombination spectra were observed by us for all GaAs/AlGaAs QW structures studied, even for those which were not intentionally doped. Thus, we relate those two peaks to the competition between the above-mentioned recombination transitions. 


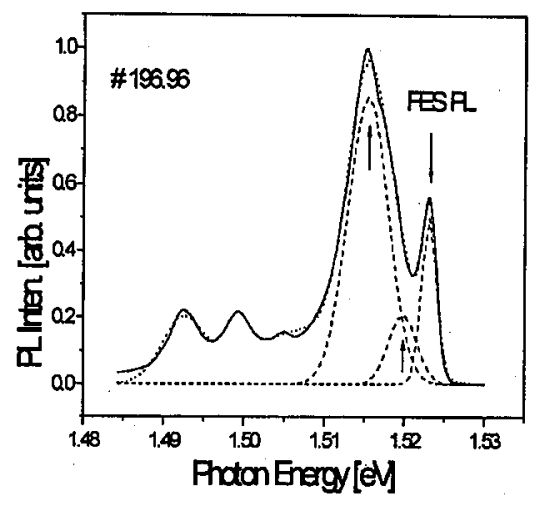

Fig. 2. Photoluminescence spectrum of the sample \#196.96 measured at 2 K. Dotted line shows the results of multi-Gaussian fit of the spectrum with 6 Gaussian peaks. Three high energy PL sub-bands are drawn with dash lines.

With increasing Be doping level a new structure appears, first as a weak shoulder (on the high energy side) of PL emission for the sample \#194.96 and then as a strong separate PL peak, at the energy $1.5230 \mathrm{eV}$, for the sample \#196.96. This PL peak, well-resolved at the highest doping level, we relate to the so-called Fermi-edge singularity emission. Additional, weak, PL peaks (three for the sample \#196.96) are observed at the low energy side of the 2D hole emission in all samples studied. PL kinetics measurements show much longer decay times for these peaks, which we attribute to the donor-acceptor pair (DAP) transitions coming likely from the GaAs substrate. Properties of these peaks will not be discussed further.

\section{Discussion of the results}

The intensive studies of $n$-type modulation doped structures indicated that two conditions should be fulfilled for the observation of 2D electron-related FES emission in the PL. Either electrons (near the Fermi edge) should be allowed to scatter resonantly to the unoccupied (e.g., $n=2$ ) sub-band [5], which results in a required admixture of $k=0$ states enhancing radiative recombination of $k \neq 0$ electrons at the Fermi edge, or they should have the possibility to recombine with the holes localized at the impurity centers or heterointerface imperfections. In the latter case the momentum conservation rule is relaxed by the hole localization. For our modulation doped $p$-type structures both conditions are likely to be fulfilled. The two-line structure of the 2D hole PL is observed suggesting some unintentional $n$-type doping of GaAs QW regions. The intensity, the spectral position (also for the two 2D hole emissions) and the energy separation between 2D hole and FES emissions clearly depend on the doping level. 2D hole emission shows a red-shift with an increasing doping level, which we relate to deepening of the notch potential at GaAs/AlGaAs interfaces and to effects of an increased screening and band gap renormalization. The latter results from the exchange interaction among free holes. In turn, the increasing energy splitting between 2D and FES free hole emission, which is equal to the Fermi energy, is a simple consequence of an increased 
concentration of $2 \mathrm{D}$ free holes $\left(p_{2 \mathrm{D}}\right)$. The band filling in $2 \mathrm{D}$, at low temperatures can be approximated by the formula

$$
\left(E_{\mathrm{F}}-E_{\mathrm{vi}}\right)=\frac{\pi \hbar^{2}}{m^{*}} p_{2 \mathrm{D}} \quad\left[\mathrm{cm}^{-2}\right] .
$$

The effective mass $m^{*}$ is now the in-plane (or transverse) effective mass $m^{\mathrm{t}}$, not to be mistaken with $m(z)$ - the mass used for calculation of energy levels. The $m^{t}$ masses are calculated from the Luttinger parameters: $\gamma_{1}=6.85, \gamma_{2}=2.1$, as: $m_{\mathrm{hh}}^{\mathrm{t}}=1 /\left(\gamma_{1}+\gamma_{2}\right)=0.112$ and $m_{\mathrm{lh}}^{\mathrm{t}}=1 /\left(\gamma_{1}-\gamma_{2}\right)=0.210$. The in-plane dispersion (for $k \rightarrow 0$ ) is assumed to be parabolic

$$
\begin{aligned}
& E_{\mathrm{hh}}(k)=-E_{\mathrm{hh}}(0)-\left(\frac{\hbar^{2}}{2 m_{0}}\right)\left[\left(\gamma_{1}+\gamma_{2}\right) k_{t}^{2}+\left(\gamma_{1}-2 \gamma_{2}\right) k_{z}^{2}\right], \\
& E_{\mathrm{lh}}(k)=-E_{\mathrm{lh}}(0)-\left(\frac{\hbar^{2}}{2 m_{0}}\right)\left[\left(\gamma_{1}-\gamma_{2}\right) k_{t}^{2}+\left(\gamma_{1}+2 \gamma_{2}\right) k_{z}^{2}\right],
\end{aligned}
$$

where $k_{t}=\sqrt{k_{x}^{2}+k_{y}^{2}}$.

For the rough estimation of band filling the valence band mixing is neglected. The accurate calculations of valence band dispersion are quite complicated and time consuming [6]. The parabolic dispersion model, despite its simplicity, reproduces at least qualitatively all essential features observed experimentally. Due to the "mass reversal" effect, the first hole subband (nominally heavy hole) is relatively fast populated. Then, the light hole subband (located $8.1 \mathrm{meV}$ higher in energy) comes into play and the rate of the shift of Fermi level with increasing carrier concentration slows down due to the higher density of states involved. The PL peak position is determined by two contradictory effects. The shift to higher energies due to the band filling and the shift to lower energies due to the exchange and correlation effects in dense hole plasma (BGR - band gap renormalization), which has been estimated after Ref. [7]. The net effect which is experimentally observed is in our case a downward shift of PL line with increasing carrier concentration, due to the prevailing effect of band gap narrowing over the band filling effect. Taking $E_{\mathrm{F}}$ as positive and BGR as negative, the peak position of $\mathrm{PL}$ line is a simple sum of the two: $E_{\mathrm{PL}}=E_{\mathrm{F}}+E_{\mathrm{BGR}}, E_{\mathrm{BGR}}<0$. The results are summarized in Table I. Note that the last two columns which list PL peak positions relative to the PL peak position in the sample \#193, calculated by outlined procedure as well as determined experimentally, show very good agreement. Fermi energies listed in Table II were obtained taking into account contributions from both the heavy hole and the light hole bands, while still retaining a parabolic approximation.

A further improvement of the agreement between the calculations and experiment should be expected by taking into account both the higher lying valence subbands (particularly for the highest doped sample \#196) and more importantly by taking into account the band mixing effects, but this is no longer a straightforward task. The trend, however, is correct. Taking into account the valence band mixing should in general increase the density of states at the energies of the onset of the light hole band and thus should decrease the calculated values of $E_{\mathrm{F}}$ and improve the agreement with experiment. 
TABLE I

Experimentally determined and calculated relative PL peak positions for the samples studied. Experimental values of Fermi energy $E_{\mathrm{F}}$ have been determined from the difference in peak positions of photoluminescence excitation (PLE) and PL spectra.

\begin{tabular}{c|c|c|c|c|c}
\hline $\begin{array}{c}\text { Sample } \\
\text { no. }\end{array}$ & $\begin{array}{c}E_{\mathrm{F}}[\mathrm{meV}]^{*} \\
\text { experimental }\end{array}$ & $\begin{array}{c}\mathrm{BGR}[\mathrm{meV}] \\
\text { theory }\end{array}$ & $\begin{array}{c}E_{\mathrm{F}}-E_{\mathrm{BGR}} \\
{[\mathrm{meV}]}\end{array}$ & $\begin{array}{c}\text { Calculated } \\
\text { PL peak } \\
\text { position } \\
\text { (with respect } \\
\text { to \#193) }\end{array}$ & $\begin{array}{c}\text { Experimental } \\
\text { PL peak } \\
\text { position } \\
\text { (with respect } \\
\text { to \#193) }\end{array}$ \\
\hline$\# 193$ & 4.48 & -18 & 13.52 & - & - \\
$\# 194$ & 6.71 & -26 & 19.29 & 5.77 & 6.0 \\
$\# 195$ & 6.71 & -28 & 21.29 & 7.77 & 7.5 \\
$\# 196$ & 10.82 & -36 & 25.18 & 11.66 & 12.0 \\
\hline
\end{tabular}

${ }^{*} \Delta E=\mathrm{PLE}-\mathrm{PL}=E_{\mathrm{F}}\left(1+m_{\mathrm{h}} / m_{\mathrm{e}}\right)=E_{\mathrm{F}}(1+0.112 / 0.0665)=2.68 E_{\mathrm{F}}$

TABLE II

Comparison of experimental and calculated Fermi energies for the samples studied.

\begin{tabular}{c|c|c|c}
\hline \hline $\begin{array}{c}\text { Sample } \\
\text { no. }\end{array}$ & $\begin{array}{c}\text { Sheet carrier } \\
\text { concentration } \\
\text { (determined } \\
\text { from SdH } \\
\text { oscillations) } \\
{\left[\mathrm{cm}^{-2}\right]}\end{array}$ & $\begin{array}{c}\text { Fermi energy } \\
E_{\mathrm{F}}[\mathrm{meV}] \\
\text { (calculated) } \\
\text { hh and lh band included } \\
\text { (parabolic approximation } \\
\text { and no band mixing) }\end{array}$ & $\begin{array}{c}E_{\mathrm{F}}[\mathrm{meV}] \\
\text { experimental } \\
\text { (determined } \\
\text { from the } \\
\text { difference }\end{array}$ \\
PLE - PL \\
\hline$\# 193$ & $4 \times 10^{11}$ & 8.25 & 4.48 \\
$\# 194$ & $8 \times 10^{11}$ & 11.37 & 6.71 \\
$\# 195$ & $10^{12}$ & 12.87 & 6.71 \\
$\# 196$ & $3 \times 10^{12}$ & 26.60 & 10.82
\end{tabular}

\section{Conclusions}

The experimentally observed features of photoluminescence and photoluminescence excitation spectra are consistently explained in terms of combined effects of: (1) band gap renormalization due to particle correlations, (2) valence band filling and (3) Fermi-edge singularity. A theoretical estimation of the influence of the above effects on the position and shape of low-temperature photoluminescence in QWs with different doping levels in the barriers are in excellent agreement with experiment. The present work is the first convincing observation of the many-body Fermi-edge singularity in the $p$-type modulation-doped AlGaAs/GaAs quantum wells. 


\section{References}

[1] M.S. Skolnick, J.M. Rorison, K.J. Nash, D.J. Mowbray, P.R. Tapster, S.J. Bass, A.D. Pitt, Phys. Rev. Lett. 58, 2130 (1987).

[2] Y.H. Zhang, N.N. Ledentsov, K. Plog, Phys. Rev. B 44, 1399 (1991).

[3] M. Bugajski, K. Regiński, M. Godlewski, M. Wesołowski, P.O. Holtz, A.V. Buyanov, B. Monemar, Acta Phys. Pol. A 90, 751 (1996).

[4] G.D. Mahan, Phys. Rev. 153, 882 (1967).

[5] J.F. Mueller, Phys. Rev. B 42, 11189 (1990).

[6] S. Łepkowski, M. Bugajski, Acta Phys. Pol. A 92, 903 (1997).

[7] S. Haacke, R. Zimmermann, D. Bimberg, H. Kal, D.E. Mars, J.N. Miller, Phys. Rev. B 45, 1736 (1992). 